\title{
Features and Outcomes of Elderly Rheumatoid Arthritis: Does the Age of Onset Matter? A Comparative Study From a Single Center in China
}

\author{
Yini Ke $\cdot$ Xiaona Dai · Danyi Xu $\cdot$ Junyu Liang $\cdot$ Ye Yu • \\ Heng Cao · Weiqian Chen · Jin Lin
}

Received: November 6, 2020 / Accepted: November 28, 2020 / Published online: December 14, 2020

(C) The Author(s) 2021

\begin{abstract}
Introduction: The aim of this work is to investigate the clinical and radiological characteristics of elderly rheumatoid arthritis and compare the outcomes between the two subgroups, elderly- and young-onset rheumatoid arthritis (EORA and YORA, respectively).

Methods: We conducted a retrospective casecontrol study on the elderly rheumatoid arthritis patients in our medical center. EORA was defined as the patient whose onset age was above 60 .

Results: A total of 142 elderly rheumatoid arthritis patients were admitted, with 79 patients in EORA and 63 in YORA group. Inflammatory parameters including C-reactive protein, D-dimer, serum ferritin, and platelet count levels were all higher in the EORA group
\end{abstract}

Supplementary Information The online version contains supplementary material available at https:// doi.org/10.1007/s40744-020-00267-8.

Y. Ke · D. Xu · J. Liang · Y. Yu · H. Cao · W. Chen $(\bowtie) \cdot J$. Lin $(\bowtie)$

Department of Rheumatology and Immunology, the First Affiliated Hospital, School of Medicine, Zhejiang University, Hangzhou, China

e-mail: cwq678@zju.edu.cn

J. Lin

e-mail: linjinzju@zju.edu.cn

X. Dai

Department of Rheumatology, Zhejiang Hospital, Hangzhou, China than those in YORA. EORA patients showed a higher score of health assessment questionnaire's disability index $(p=0.01)$ and patient global health assessment $(p=0.049)$, but a lower status of modified total sharp score $(p=0.001)$. Bivariate logistic regression analysis revealed that elderly onset of the disease (OR $2.30,95 \%$ CI [1.45-3.77]), age (OR 2.04, 95\% CI [1.22-3.41]), high disease activity (OR 1.90, 95\% CI [1.17-3.32]), and red blood cell distribution width (OR 1.81, 95\% CI [1.03-3.19]) were independent prognostic factors of disability. Age (OR 0.25, 95\% CI [0.07-0.91]), disease duration (OR 2.73, 95\% CI [0.97-7.70]), and comorbid diabetes mellitus (OR 118.10, 95\% CI [3. 50-3985.57]) independently contributed to radiographic joint damage in the elderly population. EORA patients showed increased death incidents and worse prognosis than YORA. Cox regression analysis reveals that comorbid hypertension (HR 12.02, 95\% CI [1.08-133.54]), interstitial lung disease (ILD) (HR 85.04, 95\% CI [4.11-1759.19]), and compressive fracture (HR 85.04, 95\% CI [4.11-1759.19]) are independent predictors of mortality, and that ILD (HR 50.21, 95\% CI [5.56-335.33]) and pulmonary hypertension (HR 25.37, 95\% CI [3.03-265.81]) are independent predictors of no disease remission in the EORA patients.

Conclusions: The distinct features of EORA patients make EORA a unique entity different from "classic rheumatoid arthritis". EORA patients develop an upgraded systemic 
inflammatory status, more declined life quality, and worse prognosis than the elderly YORA. Better control of the comorbidities like ILD and diabetes mellitus may benefit the management of elderly rheumatoid arthritis. Further investigation regarding the pathogenesis and therapeutic strategies of EORA is urgently warranted.

Keywords: Arthritis; Rheumatoid; Arthrography; Biomarkers; Elderly; Prognosis; Retrospective studies; Risk factors

\section{Key Summary Points}

Why carry out this study?

As the prevalence of elderly RA is increasing worldwide, the disease burden causes both health care issues and economic loss in an aging society like China.

Our study is the first to compare features of EORA and YORA among elderly patients, and to carry out prognostic predictions in China.

What was learned from the study?

EORA patients showed distinct features from the elderly YORA, and the older onset age of the disease really matters to predict a worse outcome.

Based on the poorer performance of EORA patients at baseline and during the followup, more aggressive treatment is needed to reach better disease remission.

Treatment of comorbidities may benefit the survival and help restore life quality for elderly patients, especially EORA patients.

\section{DIGITAL FEATURES}

This article is published with digital features, including a summary slide, to facilitate understanding of the article. To view digital features for this article go to https://doi.org/10. 6084/m9.figshare.13295975.

\section{INTRODUCTION}

Rheumatoid arthritis (RA) is one of the most common inflammatory autoimmune diseases, characterized by symmetrical polyarthritis and progressive destruction of joints. RA commonly affects patients aged $30-50$ years old [1], while in those aged above 60 , the disease is called elderly RA. Elderly RA patients can be classified into two clinical subsets, elderly onset RA (EORA) and young-onset elderly RA (elderly YORA, eYORA). EORA, also termed late-onset RA, is defined as RA with an onset age over 60, which accounts for approximately $10-33 \%$ of the elderly RA cases [2, 3]. Recently, special attention has been paid to the novelty of EORA in terms of its genetic predisposition, clinical features, and therapeutic options [4]. It is reported that EORA is associated with HLADRB1*01, * $13,{ }^{*} 14$, while its younger counterpart, the YORA, exhibits an increased frequency of $\mathrm{DRB} 1{ }^{*} 04$, which indicates younger onset age and radiological erosions of RA [5]. In contrast to typical RA, EORA seems to present with a more abrupt disease onset, greater alteration in life quality, and a better response to biological agents [6-8]. However, some studies found that EORA may have a more benign form of disease than YORA $[9,10]$.

It is estimated that approximately $2-9 \%$ of the world's population is elderly, and the prevalence is still climbing in an aging society like China. Elderly RA patients are always associated with cognitive impairment, depression, and frailty [11]. The atypical manifestations of EORA, such as fever, myalgia, anemia, and polymyalgia rheumatica (PMR)-like syndrome increase the difficulties in the diagnosis of diseases [12-15].Higher incidence of co-morbidities and drug-related adverse effects in elderly patients also raise therapeutic challenges to better remission and disease management $[7,16]$, although the outcome of these two disease subtypes is intensively studied and compared. 
Until now, there have been several crosssectional and a few prospective studies investigating the features of EORA patients $[2,9,12]$. However, the ways EORA differs from elderly YORA remains unclear. In this retrospective case-control study, we included a cohort of elderly RA patients and described features of EORA patients. In addition, we identified the risk factors that contribute to the disability and radiographic damage in the elderly, and predictors of disease progression and mortality during follow-up, aiming to provide better understanding of the disease affecting the elderly population. Our study investigates the comparative features of EORA and YORA among the elderly, and carries out prognostic predictions in China. We believe that more aggressive treatment is needed to reach better disease remission and treatment of comorbidities may benefit survival and help restore life quality of elderly patients, especially EORA patients.

\section{METHODS}

\section{Study Design}

As a retrospective study, subjects were selected from patients who were hospitalized in the Department of Rheumatology and Immunology, the First Affiliated Hospital, School of Medicine, Zhejiang University (FAHZJU) China from January 2013 to July 2017. Subjects' baseline data collection was conducted from September 10 to October 10, 2017. Inclusion criteria: (1) Fulfilled the 2010 American College of Rheumatology/European League Against Rheumatism collaborative initiative revised Rheumatoid Arthritis Classification Criteria; (2) Aged above 60 years old. EORA was defined as an RA patient whose disease onset age was more than 60 years old while that of YORA was between 18 and 59 years old. Exclusion criteria: (1) Patients with acute infectious diseases; (2) Trauma; (3) Chronic conditions such as malignancy, severe congestive heart failure, Parkinson's disease, and cognitive disorders. Patients were followed from the day of hospitalization until time of the events including interstitial lung disease (ILD), pulmonary arterial hypertension $(\mathrm{PAH})$, death, or end of the follow-up (maximum 24 months), which ever came first. Patients who were lost to follow-up without death from any cause within 6 months after hospitalization were also excluded. The protocol was approved by the institutional ethic review board at the First Affiliated Hospital, School of Medicine, Zhejiang University (Reference Number: 2017-648) and conducted in accordance with the Declaration of Helsinki. Written consent was obtained from the patients at the first day of hospitalization for their date being stored in our dataset and used for scientific research.

General information, clinical symptoms, comorbid conditions, serum parameters such as blood routine, biochemistry, thyroid function (total triiodothyronine, free thyroxine, and thyrotropin), bone turnover markers (type I collagen amino-terminal propeptide, N-terminal osteocalcin, parathormone and 25-hydroxyvitamin D), metabolic parameters (triglyceride, cholesterol, uric acid) and treatment records were retrospectively collected. Radiological profiles were reviewed by the X-ray film of both hands and assessed using modified total Sharp score (mTSS). Serum parameters were measured simultaneously on the first day of hospitalization.

\section{Statistical Analysis}

Statistical analysis was performed using SPSS software version 23.0 (IBM Corporation, Armonk, NY, USA). Continuous variables were expressed as a median value with (Q1-Q3). The Kolmogorov-Smirnov test was used to detect whether continuous data were normally distributed. Mann-Whitney $U$ test was adopted for comparisons of continuous data. Chi-squared test was applied to carry out the comparisons of categorical variables. Binary logistic regression analysis was conducted to identify risk factors of EORA. Spearman's correlation and Kendall rank correlation coefficients were calculated for bivariate relationships. Survival curves were estimated using the Kaplan-Meier method. Univariate and multivariate cox proportional hazards regression analyses were subsequently 
adopted to identify the influence of factors on the time to death from any cause in EORA patients. All tests were two-sided, and $p<0.05$ was considered statistically significant.

\section{RESULTS}

\section{Demographic and Clinical Characteristics}

At baseline, a total of 142 elderly RA patients were admitted, including 79 patients in the EORA and 63 in YORA group. Demographic and clinical characteristics of the subjects are shown in Table 1.

The median age of patients was 67.0 years (62.0-72.0) in the whole subjects, 70.0 years (66.0-74.0) in the EORA group, and 63.0 years (61.0-68.0) in YORA group when admitted. The EORA group had a shorter disease duration than the YORA group (0.6 vs. 11.0 years, $p<0.001$ ). The proportion of female patients was lower in the EORA group than in the YORA group (58.2 vs. $79.4 \%, p=0.011)$. There were more smokers identified in EORA group than YORA (36.7 vs. $19.0 \%, p=0.026$ ).

Clinically, large joints including shoulder, elbow, and knees were more commonly affected in the EORA group at the disease onset (34.2 vs. $13.6 \%, p<0.006)$. Patients with EORA showed significantly higher health assessment questionnaire's disability index (HAQ-DI) score (1.3 vs. 0.67, $p=0.01$ ), and higher level of global health (GH) assessment (30.0 vs. 20.0, $p=0.049$ ) recorded on a visual analogue scale. Furthermore, we found that C-reactive protein (CRP) level detected in EORA patients was also significantly higher than that in the YORA group $(36.1 \mathrm{mg} / \mathrm{l}$ vs. $18.2 \mathrm{mg} / \mathrm{l}, p=0.010)$. Disease Activity Score of 28 joints (DAS28) calculated by erythrocyte sedimentation rate (ESR) and CRP tended to be higher in the EORA group, with $p=0.066$ and 0.071 , respectively. In addition, the modified total Sharp score

Table 1 Demographic and baseline clinical features of patients with EORA and YORA

\begin{tabular}{lllr}
\hline Variables & EORA $(\boldsymbol{n}=\mathbf{7 9})$ & YORA $(\boldsymbol{n}=\mathbf{6 3})$ & $\boldsymbol{p}_{\text {trend }}$ value \\
\hline Female sex (\%) & $46(58.2)$ & $50(79.4)$ & 0.011 \\
Age, years & $70.0(66.0-74.0)$ & $63.0(61.0-68.0)$ & $<0.001$ \\
Disease duration, years & $0.6(0.3-3.0)$ & $11.0(7.0-20.0)$ & $<0.001$ \\
BMI, kg/m & $20.8(18.8-22.9)$ & $20.7(18.9-24.0)$ & 0.422 \\
Cigarette smoking (\%) & $29(36.7)$ & $12(19.0)$ & 0.026 \\
Large joints onset (\%) & $27(34.2)$ & $9(14.2)$ & 0.007 \\
Morning stiffness (\%) & $64(81.0)$ & $52(82.5)$ & 0.829 \\
HAQ-DI & $1.3(0.7-2.0)$ & $0.67(0.3-1.3)$ & 0.019 \\
DAS28-ESR (4) & $5.67(4.6-6.0)$ & $5.5(4.1-6.5)$ & 0.066 \\
mTSS & $50.0(36.0-42.0)$ & $118.0(27.8-156.0)$ & 0.001 \\
JSN score & $18.0(13.0-18.0)$ & $57.5(17.8-71.0)$ & 0.006 \\
Erosion score & $32.0(23.0-46.0)$ & $62.5(9.5-87.5)$ & 0.001 \\
\hline
\end{tabular}

DAS28-ESR 28-joint disease activity score using ESR, EORA elderly-onset rheumatoid arthritis, ESR erythrocyte sedimentation rate, $G H$ global health, $H A Q-D I$ health assessment questionnaire's disability index, JSN joint space narrowing, mTSS modified total Sharp score, SJC swollen joint count, TJC tender joint count, YORA young-onset rheumatoid arthritis. The variable is expressed as median (Q1-Q3). Mann-Whitney $U$ test was for comparison of continuous data. Chisquared test was for categorical variables 
(mTSS), together with the erosion score and joint space narrowing score, were all significantly lower in the EORA group. The above results indicate that EORA patients differ from YORA in multiple demographic aspects and that they present a worse physical and functional assessment, and a higher disease activity during hospitalization.

\section{Serological Characteristics}

We conducted a comprehensive comparison of the serological features between the two groups. Serum parameters including inflammatory factors, autoimmune antibodies, complete blood cell count, biochemical analysis, and bone metabolism markers, and the results were listed and compared. As was shown in Table 2, when the autoimmune antibodies were taken into account, EORA had fewer patients with high titer (defined as tripled-titer) of anti-CCP (82.5 vs. $66.2 \%, p=0.035$ ). However, the levels of rheumatoid factor (RF), anti-mutated citrullinated vimentin antibody (anti-MCV), or anticyclic citrullinated peptide antibody (anti-CCP) were similar between the two groups. Interestingly, the proportion of ANA positivity was $29.9 \%$ in EORA patients, lower than that of YORA $(49.2 \%, p=0.023)$. As the secondary Sjögren's syndrome (sSS) is a common rheumatic disease co-morbid with RA, which is characterized by ANA sero-positive, we subsequently ruled out the cases of sSS. However, we can still detect the statistical difference of ANA between the two groups $(p=0.038)$. Antimyeloperoxidase (MPO) antibody was significantly lower in the EORA group $(p=0.042)$. The above results indicated potentially novel pathogenesis of EORA.

As for the inflammatory parameters, higher levels of D-dimer (2411.5 vs. $1829.0 \mu \mathrm{g} / \mathrm{l}$, $p=0.048)$, ferritin level $(360.1 \mathrm{ng} / \mathrm{ml}$ vs.

Table 2 Serological characteristics of EORA and YORA patients

\begin{tabular}{lllr}
\hline Parameters & EORA & YORA & $p$ value \\
\hline RF (high titer) (\%) & $49(64.5)$ & $42(66.7)$ & 0.859 \\
Anti-CCP (high titer) (\%) & $51(66.2)$ & $52(82.5)$ & 0.035 \\
Anti-MCV (high titer) (\%) & $16(59.3)$ & $9(64.3)$ & $>0.999$ \\
ANA positive (> 1:20) (\%) & $23(29.9)$ & $30(49.2)$ & 0.023 \\
MPO, U/ml & $0.90(0.6-1.3)$ & $1.05(0.6-1.6)$ & 0.042 \\
ESR, mm/h & $74.0(34.8-90.0)$ & $72.0(31.0-92.0)$ & 0.552 \\
CRP, mg/l & $36.1(16.0-78.8)$ & $18.2(7.5-50.4)$ & 0.010 \\
Ferritin, ng/ml & $360.1(219.0-576.8)$ & $206.4(138.2-367.7)$ & 0.001 \\
D-dimer, ug/l & $2411.5(1147.3-4856.0)$ & $1829.0(949.5-3509.5)$ & 0.048 \\
Platelet count, 10E9/1 & $286.5(230.0-344.0)$ & $243.0(172.0-304.0)$ & 0.012 \\
IgA, mg/dl & $324.0(242.5-398.5)$ & $380.0(260.3-473.8)$ & 0.040 \\
IgG, mg/dl & $1420.0(1066.5-1732.0)$ & $1588.5(1092.5-1918.5)$ & 0.058 \\
IgM, mg/dl & $127.0(85.1-201.5)$ & $131.0(90.1-183.3)$ & 0.990 \\
\hline
\end{tabular}

$A N A$ Anti-nuclear antibody, $A N C A$ antineutrophil cytoplasmic antibody, anti-CCP anti-cyclic citrullinated peptide antibody, anti-MCV anti-mutated citrullinated vimentin antibody, $\operatorname{Ig} A$ immunoglobulin A, $\operatorname{Ig} G$ immunoglobulin $\mathrm{G}, \operatorname{Ig} M$ immunoglobulin $\mathrm{M}, M P O$ anti-myeloperoxidase antibody

The variable is expressed as median (Q1-Q3). Mann-Whitney $U$ test was for comparison of continuous data. Chi-squared test was for categorical variables 
$206.4 \mathrm{ng} / \mathrm{ml}, \quad p<0.001) \quad$ platelet count $\left(286.5^{*} 10 \mathrm{E} 9 / 1\right.$ vs. $\left.243.0^{*} 10 \mathrm{E} 9 / \mathrm{l}, p=0.012\right)$ was found in EORA group. Together with the results mentioned above, we could postulate that there may be a more active systemic inflammatory environment in EORA patients. Furthermore, the change value of CRP after a course of therapy was greater in EORA group $(p=0.046)$, which might predict a better response of EORA patients to therapy. Meanwhile, we found that hemoglobin, thyroid hormone, bone turnover markers, and metabolic parameters were similar in the two groups.

\section{Comorbidities and Medication History}

Medical history of patients was carefully reviewed in both groups. The related comorbidities included metabolic diseases, thromboembolic diseases, orthopedic complications, and importantly, rheumatic diseases. ILD, secondary Sjögren's syndrome (SS), and symmetrical synovitis with pitting edema (RS3PE) were all recorded. The incident rates of the comorbidities in both groups are listed in Supplementary Table 1 .

The incident rate of RS3PE was $10.1 \%$ in EORA group, while no RS3PE patients were identified in YORA group $(p=0.008)$. More patients in YORA group were identified with leukocytopenia ( 8.5 vs. $1.4 \%, p=0.023)$. However, when the cases of co-morbid sSS were excluded, the statistical difference was no longer detected $(p=0.09)$. The incident rates of ILD, sSS, cardiovascular disease, type 2 diabetes mellitus (T2DM), osteoporosis, and intermuscular thrombosis were similar between the two groups. Medication history including treatment with conventional disease-modifying antirheumatic drugs (DMARDs), analgesics, and glucocorticoids were reviewed in the two groups. The prescription of non-steroidal antiinflammatory drugs (NSAIDs) was more common in the EORA group $(p=0.007)$. However, no statistically significant difference was detected in the utilization of DMARDs, or glucocorticoids between the two groups. Details are shown in Supplementary Table 2.

\section{Impact Factors of EORA}

In the binary logistic regression analysis, we adopted the significant demographic, clinical, and serological parameters listed above. We found out that large joint onset [odds ratio (OR) $3.41,95 \%$ confidence interval (CI) [1.39-8.39]], female gender (OR 2.86, 95\% CI [1.17-6.88]), WBC (OR 1.35, 95\% CI [1.22-3.41]) and higher level of CRP (OR 1.02, 95\% CI [1.00-1.03]) were the positively associated with developing EORA, while higher titer of serum ACPA (OR 0.30, 95\% CI [0.12-0.74]) was with YORA. Details are listed in Table 3.

\section{Disability Status}

The HAQDI score is an indicator of current disability. After calculating the binary Spearman and Kendall correlation coefficients, we found that in our cohort, HAQ-DI was positively correlated with the early onset of the disease, onset age of the disease, disease activity, levels of inflammatory parameters including ESR, CRP, and D-dimer, red blood cell distribution width, but was negatively correlated with the level of WBC, hemoglobin, and IL-6. Details were shown in Supplementary Table 3.

Bivariate logistic regression analysis revealed the association between disability (HAQDI $>1$ ) and various variables such as elderly onset (OR 2.30, 95\% CI [1.45-3.77]) age (interval of every 10 years) (OR 2.04, 95\% CI [1.22-3.41]), high disease activity (OR 1.90, 95\% CI [1.17-3.32]), red blood cell distribution width (RDW) (OR 1.81, 95\% CI [1.03-3.19]) and albumin level (OR 0.53, 95\% CI [0.29-0.96]) (Table 4).

\section{Radiological Features}

As mentioned above, the EORA group showed a much lower level of modified total sharp score, indicating patients with EORA exhibited lighter joint damage than YORA.

Furthermore, we conducted the binary Spearman and Kendall correlation to find out the association between mTSS and other features of elderly RA. We found that mTSS was positively correlated with the disease duration, 
Table 3 Logistic regression analysis of impact factors in EORA patients

\begin{tabular}{llllll}
\hline Variables & $\boldsymbol{P}$ value & SE & OR & \multicolumn{2}{l}{$\mathbf{9 5 \% \text { CI for OR }}$} \\
\hline Large joint onset & 0.007 & 0.459 & 3.41 & 1.39 & 8.39 \\
Female & 0.011 & 0.423 & 2.86 & 1.17 & 6.88 \\
WBC & 0.001 & 0.081 & 1.35 & 1.15 & 1.58 \\
CRP & 0.006 & 0.006 & 5.72 & 2.88 & 7.39 \\
Anti-CCP (high titer) & 0.010 & 0.469 & 0.30 & 0.12 & 0.74 \\
Constant & 0.007 & 0.685 & 0.16 & & \\
\hline
\end{tabular}

anti-CCP anti-cyclic citrullinated peptide antibody, $C I$ confidence interval, $C R P$ c-reactive protein, $O R$ odds ratio, $S E$ standard error, $W B C$ white blood cell count

Table 4 Bivariate logistic regression analysis of the impact factors on disability

\begin{tabular}{llllll}
\hline Variables & $\boldsymbol{P}$ value & SE & OR & \multicolumn{2}{l}{$\mathbf{9 5 \%}$ CI for OR } \\
\hline Elderly disease onset & 0.008 & 0.263 & 2.30 & 1.45 & 3.77 \\
Age (every 10 years) & 0.007 & 0.456 & 2.04 & 1.22 & 3.41 \\
DAS28-ESR & 0.015 & 0.317 & 1.90 & 1.17 & 3.32 \\
Morning stiffness & 0.033 & 0.247 & 1.88 & 1.01 & 3.24 \\
Albumin & 0.036 & 0.305 & 0.53 & 0.29 & 0.96 \\
RDW & 0.040 & 0.283 & 1.81 & 1.03 & 3.19 \\
Constant & 0.004 & 0.510 & 0.158 & & \\
\hline
\end{tabular}

$D A S 28$ 28-joint disease activity score, $R D W$ red blood cell distribution width

the RDW level, comorbid osteoporosis and T2DM, and inflammatory indexes like ferritin levels. Interestingly, a significant correlation was identified in renal function index including estimated glomerular filtration rate (eGFR), serum creatinine, urine erythrocyte, and urine protein. On the contrary, mTSS was shown to be negatively correlated with early onset of the disease, female gender, onset age, body mass index, and hemoglobin level, however, the relationship between mTSS and disease activity was not detected. Details are shown in Supplementary Table 3.

Bivariate logistic regression analysis revealed a significant association between mTSS and variables such as age (interval of every 10 years) (OR 0.25, 95\% CI) 0.07-0.91]), RDW (OR 8.24,
95\% CI [2.22-30.6]), and disease duration (OR 2.73, 95\% CI [0.97-7.70]). Comorbidities as type 2 diabetes mellitus (T2DM) (OR 118.10, 95\% CI [3. 50-3985.57]) was also associated with mTSS (Table 5).

\section{Follow-Up}

In prognostic study, a total of 88 patients were followed, with 45 patients in EORA and 43 in YORA. Patients were followed from the day of hospitalization. The outcomes were incident comorbidities and all-cause mortality until 24 months after hospitalization. In the EORA group, 11 deaths were observed during followup. The all-cause mortality was $24.4 \%(11 / 45)$ in EORA group, significantly higher than that of 
Table 5 Bivariate logistic regression analysis of the impact factors on mTSS

\begin{tabular}{lllcrr}
\hline Variables & Sig & SE & OR & \multicolumn{2}{l}{ 95\% CI for OR } \\
\hline Age (every 10 years) & 0.036 & 0.668 & 0.25 & 0.07 & 0.91 \\
RDW & 0.002 & 0.669 & 8.24 & 2.22 & 30.60 \\
Disease duration & 0.049 & 0.528 & 2.73 & 0.97 & 7.70 \\
T2DM & 0.008 & 1.796 & 118.07 & 3.50 & 3985.57 \\
Constant & 0.016 & 1.012 & 0.087 & & \\
\hline
\end{tabular}

$R D W$ red blood cell distribution width, T2DM type 2 diabetes mellitus

the YORA $6.9 \%(3 / 43), p=0.039$. Details of comparison between the two groups during follow-up is shown in Supplementary Table 4. Survival curves estimated by Kaplan-Meier method revealed a higher death rate in EORA, with 47.28 (28.62-68.70) months median survival period in EORA and 168.50 (93.90-283.57) months in YORA (see Fig. 1).

\section{Risk Factors for Mortality Outcomes}

Univariant Cox regression analysis of mortality in EORA patients during follow-up revealed that comorbid hypertension, pulmonary arterial hypertension, gout, ILD, and compression fractures increased the incident death in EORA patients. Further multivariant Cox regression

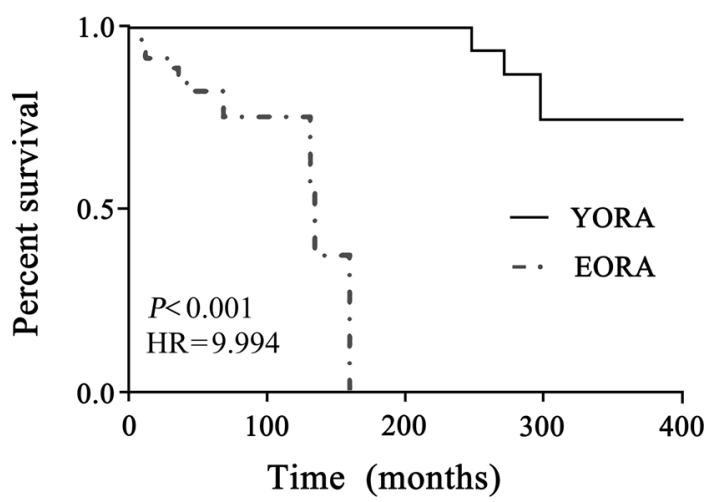

Fig. 1 Survival curves stratified by disease onset of elderly RA patients. Survival curves are estimated by Kaplan-Meier method. The media survival period is 47.28 (28.62-68.70) months in EORA and is 168.50 (93.90-283.57) months in YORA analysis was conducted based on the univariant Cox regression. It was found that hypertension $(\mathrm{HR}=12.02,95 \%$ CI [1.08-133.54], $p=0.043)$, ILD $\quad(\mathrm{HR}=85.04, \quad 95 \%$ CI $[4.11-1759.19]$, $p=0.004)$ and compressive fracture (HR = 65.07, 95\% CI [2.15-1971.10], $p=0.016)$ were independently associated with mortality in EORA patients.

\section{Predictors for No Disease Remission}

No statistically significant difference was detected in remission rates between the two groups (24.4 vs. 32.6\%, $p=0.399)$. Disease remission was defined as DAS $28<2.6$. Univariant Cox regression analysis was also performed to predict risk factors of the no remission (NR) in EORA patients. Higher baseline DAS28 level, large joint onset, comorbid hypertension, diabetes mellitus, ILD and PAH are associated with increased risk for NR in EORA patients. Multivariant analysis showed that comorbid ILD (HR $=50.21, \quad 95 \%$ CI [5.56-335.33], $p=0.001)$ and PAH $(\mathrm{HR}=25.37$, 95\% CI [3.03-265.81], $p=0.003$ ) were the independent predictors of NR in EORA patients.

\section{DISCUSSION}

We conducted a case-control study to describe the clinical, serological, and radiological characteristics of elderly rheumatoid arthritis stratified by the onset age of the disease. In addition, logistic regression and Cox regression analysis were conducted to explore the risk factors of 
disease outcomes. We believe that the onset age of RA matters among the elderly population and that EORA is a unique entity different from "classic rheumatoid arthritis". EORA patients develop an upgraded systemic inflammatory status, more declined physical and functional assessment and worse prognosis than the elderly YORA patients. The comorbidities like ILD and diabetes mellitus contribute to no disease remission and mortality. Although our study is not the first to address the characteristics of EORA and YORA, to our knowledge, it is the first to investigate the features among the elderly, and to carry out prognosis predictions in this region.

In our study of the elderly RA, a lower proportion of female in EORA group was observed, which is consistent with previous reports [9]. Similarly, we found that large joints were more commonly affected in the EORA group at disease onset, making it more easily for EORA patients to be misdiagnosed as scapulohumeral periarthritis or osteoarthritis in clinical practice $[9,17,18]$. Although we did not observe statistically significant differences in disease activity between the two groups, DAS 28 calculated by ESR/CRP tended to be higher in the EORA group. The poorer performance of HAQ-DI score and patients' assessments of global health indicating a greater decline in life quality for EORA patients [2]. Furthermore, a higher level of nonspecific inflammatory parameters including CRP, D-dimer, ferritin, and platelet count is observed in our study, and it is thus reasonable to speculate that EORA patients develop a more active systemic inflammation during the course among the elderly RA patients [19].

RA patients ultimately end up with bone destruction and cartilage changes within the first few years after disease onset. In our study, logistic regression showed T2DM were independent risk factors of bone destruction in the elderly population. Previous studies have reported that T2DM patients are at increased risk of developing RA and that there is a direct correlation between the degree of impaired glucose and the severity of the inflammatory status in patients with RA. In recent decades, much evidence has shown that there is a potent interaction between glucose levels and bone metabolism, and that diabetes mellitus increased disruption of microstructure and fragility of bone $[20,21]$. Thus, we believed that aggressive control of the comorbidities such as T2DM may be beneficial to reduces the progression of bone destruction in elderly patients.

During follow-up, we found that survival rates of EORA patients were significantly lower than that of the elderly YORA patients. The Cox regression model revealed that hypertension, ILD, and compressive fractures were independently associated with higher mortality and that ILD and PAH were predictors of no disease remission in EORA. We did not observe a correlation between the seropositivity and higher death incidents that have been reported previously. Hypertension is a risk factor of CVD in RA patients, and, untreated hypertension contributes to the subclinical CVD and impact renal function, which will lead to adverse events including death in RA patients [22]. As a common co-morbidity of RA, ILD is more prevalent in older patients. ILD is also a predictor of poor prognosis in RA, and the median survival period is 3 years in RA-ILD patients $[23,24]$. Thus, routine high-resolution CT is recommended during the follow-up of the EORA [23].

It has been widely accepted that the treatment of EORA pursues the same objectives as in younger patients: to control the clinical manifestations, prevent structural damage, restore function, and decrease excessive mortality [25-27]. In our study, other than NSAIDs, no statistical differences in medications history were detected between the two groups, which was consistent with previous studies. Tutuncu and his colleagues [8] reported that EORA patients always receive less treatment than YORA, despite an identical duration of disease, comparable activity, and severity. The above results make us reconsider the treating strategy of EORA patients: the treatment of comorbidities such as ILD and PAH will contribute to disease remission and restore life quality of the EORA patients, making it an indispensable aspect of the treat-to-target approach [28].

We did not observe higher incident rates of morbidities such as CVD, hypertension, 
diabetes mellitus, or thyroid disease in the EORA group, as described by other studies $[12,29,30]$. We speculate that in most of the previous studies, the observed age of patients of YORA ranged from 18 to 59 years old [31, 32], but not $>60$ years as in our cohort. As the CVD, diabetes mellitus, and hypertension are the diseases closely related to aging, it is reasonable to identify that two groups share similar comorbidity spectrum in elderly patients.

Red blood cell distribution width is a parameter of the complete blood count test, which reflects the variability in the size of circulating erythrocytes and is used for differential diagnosis of anemia. In our study, we found that RDW was an independent factor of both higher HAQ-DI and mTSS. In recent years, intensive studies about RDW reveal that it is a marker of chronic inflammation and oxidative stress and that RDW is associated with various disease and statuses including aging, chronic inflammatory diseases, and overall mortality in the general population. In a multiethnic large population study, Arbel et al. reported that subjects with RDW $>17 \%$ had a substantially higher risk of all-cause mortality compared to those with RDW $<13 \%$ after multiple adjustments [33, 34]. Actually, in our study, after plotting the receiver operating characteristic curve, we found that the area under the curve (AUC) for RDW to predict disability was 0.670 at an optimal cut-off level of 14.1\%; and AUC for RDW to predict radiographic damage was 0.800 at an optimal cut-off level of $14.2 \%$ (data not shown). We hereby believe that RDW may be an important inflammatory and prognostic marker, and that RDW $>14 \%$ may be a cut-off value to predict the disability and joint damage for elderly RA patients.

The most significant limitations of this study were the retrospective and observational nature as well as the small sample size. Lack of the healthy elderly controls might cause overestimation of the quality of the tests in disease. Thus, large-scale, multi-centered prospective trials are needed to confirm the results of this study in the future. Secondly, in the EORA group, median disease duration is 0.6 years, which means that a big proportion of EORA patients in our cohort would be newly onset RA
(NORA, disease duration less than 12 months). It is reasonable to believe that YORA patients are more likely to receive a long course of treatment and that disease activity at RA diagnosis and post-treatment may be different $[35,36]$, but we could take NORA patients a bias in our analysis. Lastly but not least, follow-up of the radiographic progression is of great importance and needed to be included in our future studies.

\section{CONCLUSIONS}

As the prevalence of elderly RA is increasing worldwide, the disease burden causes both health care issues and economic loss in an aging society like China. EORA presents with distinct features from the younger onset entity, including a more active inflammatory state, greater loss of life quality, and higher death incidents. Further investigation into the pathogenesis and management of EORA is urgently warranted.

\section{ACKNOWLEDGEMENTS}

We thank all the participants in the study.

Funding. This work was supported by the National Natural Science Foundation of China (Award Number: 81701600, Grant recipient Weiqian Chen) and Natural Science Foundation of Zhejiang Province (Award Number: LQ20H100003, Grant Recipient Yini Ke; Award Number: LQ17H100001, Grant Recipient Weiqian Chen; Award Number: LGF18H100001, Grant Recipient Jin Lin). The transportation fee, purchase of the SPSS software, and the Rapid Service Fee were funded by the sponsors mentioned above.

Authorship. All named authors Yini Ke, Xiaona Dai, Danyi $\mathrm{Xu}$, Junyu Liang, Ye $\mathrm{Yu}$, Heng Cao, Weiqian Chen, and Jin Lin meet the International Committee of Medical Journal Editors (ICMJE) criteria for authorship for this article, take responsibility for the integrity of the work as a whole, and have given their approval for this version to be published. 
Disclosures. The authors Yini Ke, Xiaona Dai, Danyi Xu, Junyu Liang, Ye Yu, Heng Cao, Weiqian Chen, and Jin Lin declare that the research was conducted in the absence of any personal, financial, commercial, or academic relationships that could be construed as a potential conflict of interest.

Compliance with Ethics Guidelines. The protocol was approved by the institutional ethic review board at the First Affiliated Hospital, School of Medicine, Zhejiang University (Reference Number: 2017-648) and conducted in accordance with the Declaration of Helsinki. The written consent was obtained from patients at the first day of hospitalization for their date being stored in our dataset and used for scientific research.

Data Availability. The datasets generated during and/or analyzed during the current study are available from the corresponding author on reasonable request. Readers are welcome to contact linjinzju@zju.edu.cn to request the data.

Open Access. This article is licensed under a Creative Commons Attribution-NonCommercial 4.0 International License, which permits any non-commercial use, sharing, adaptation, distribution and reproduction in any medium or format, as long as you give appropriate credit to the original author(s) and the source, provide a link to the Creative Commons licence, and indicate if changes were made. The images or other third party material in this article are included in the article's Creative Commons licence, unless indicated otherwise in a credit line to the material. If material is not included in the article's Creative Commons licence and your intended use is not permitted by statutory regulation or exceeds the permitted use, you will need to obtain permission directly from the copyright holder. To view a copy of this licence, visit http://creativecommons.org/licenses/by$\mathrm{nc} / 4.0 /$.

\section{REFERENCES}

1. Olofsson T, Petersson IF, Eriksson JK, et al. Predictors of work disability after start of anti-TNF therapy in a national cohort of Swedish patients with rheumatoid arthritis: does early anti-TNF therapy bring patients back to work? Ann Rheum Dis. 2017;76:1245-52.

2. Krams T, Ruyssen-Witrand A, Nigon D, et al. Effect of age at rheumatoid arthritis onset on clinical, radiographic, and functional outcomes: the ESPOIR cohort. Jt Bone Spine. 2016;83:511-5.

3. Deal CL, Meenan RF, Goldenberg DL, et al. The clinical-features of elderly-onset rheumatoidarthritis-a comparison with younger-onset disease of similar duration. Arthritis Rheum. 1985;28: 987-94.

4. Kobak S, Bes C. An autumn tale: geriatric rheumatoid arthritis. Ther Adv Musculoskelet Dis. 2018;10: 3-11.

5. Villa-Blanco JI, Calvo-Alen J. Elderly onset rheumatoid arthritis: differential diagnosis and choice of first-line and subsequent therapy. Drugs Aging. 2009;26:739-50.

6. Olivieri I, Palazzi C, Peruz G, Padula A. Management issues with elderly-onset rheumatoid arthritis-an update. Drugs Aging. 2005;22:809-22.

7. Villa-Blanco JI, Calvo-Alen J. Elderly onset rheumatoid arthritis differential diagnosis and choice of first-line and subsequent therapy. Drugs Aging. 2009;26:739-50.

8. Tutuncu Z, Kavanaugh A. Rheumatic disease in the elderly: rheumatoid arthritis. Clin Geriatr Med. 2005;21:513.

9. Tan TC, Gao X, Thong BY, et al. Comparison of elderly- and young-onset rheumatoid arthritis in an Asian cohort. Int J Rheumat Dis. 2017;20:737-45.

10. Mueller RB, Kaegi T, Finckh A, et al. Is radiographic progression of late-onset rheumatoid arthritis different from young-onset rheumatoid arthritis? Results from the Swiss prospective observational cohort. Rheumatology (Oxford, England). 2014;53: 671-7.

11. Xu X, Li QJ, Xia S, Wang MM, Ji W. Tripterygium glycosides for treating late-onset rheumatoid arthritis: a systematic review and meta-analysis. Altern Ther Health Med. 2016;22:32-9.

12. Spinel-Bejarano N, Quintana G, Heredia R, et al. Comparative study of elderly-onset rheumatoid arthritis and young-onset rheumatoid arthritis in a 
Colombian population: clinical, laboratory and HLA-DRB1 findings. Clin Exp Rheumatol. 2013;31: 40-6.

13. Bajocchi G, La Corte R, Locaputo A, Govoni M, Trotta F. Elderly onset rheumatoid arthritis: clinical aspects. Clin Exp Rheumatol. 2000;18:S49-50.

14. Arnold MB, Bykerk VP, Boire G, et al. Are there differences between young- and older-onset early inflammatory arthritis and do these impact outcomes? An analysis from the CATCH cohort. Rheumatology. 2014;53:1075-86.

15. Wakura D, Kotani T, Takeuchi T, et al. Differentiation between polymyalgia rheumatica (PMR) and elderly-onset rheumatoid arthritis using 18F-fluorodeoxyglucose positron emission tomography/computed tomography: is enthesitis a new pathological lesion in PMR? PLoS ONE. 2016;11: e0158509.

16. Leon L, Gomez A, Vadillo C, et al. Severe adverse drug reactions to biological disease-modifying antirheumatic drugs in elderly patients with rheumatoid arthritis in clinical practice. Clin Exp Rheumatol. 2018;36:29-35.

17. Turkcapar N, Demir O, Atli T, et al. Late onset rheumatoid arthritis: clinical and laboratory comparisons with younger onset patients. Arch Gerontol Geriatr. 2006;42:225-31.

18. Mine T, Ihara K, Kawamura H, Kuriyama R, Date R. Knee arthritis without other joint symptoms in the elderly with seronegative elderly onset rheumatoid arthritis. Open Orthopaedics J. 2016;10:793-6.

19. Sugihara T, Harigai M. Targeting low disease activity in elderly-onset rheumatoid arthritis: current and future roles of biological disease-modifying antirheumatic drugs. Drugs Aging. 2016;33:97-107.

20. Napoli N, Chandran M, Pierroz DD, et al. Mechanisms of diabetes mellitus-induced bone fragility. Nat Rev Endocrinol. 2017;13:208-19.

21. Hofbauer LC, Lecka-Czernik B, Seibel MJ. Sweet and brittle-diabetes mellitus and the skeleton. Bone. 2016;82:1.

22. Midtbo H, Gerdts E, Kvien TK, et al. The association of hypertension with asymptomatic cardiovascular organ damage in rheumatoid arthritis. Blood Press. 2016;25:298-304.

23. Kim HC, Lee JS, Lee EY, et al. Risk prediction model in rheumatoid arthritis-associated interstitial lung disease. Respirology. 2020;2:2.

24. Koduri G, Norton S, Young A, et al. Interstitial lung disease has a poor prognosis in rheumatoid arthritis: results from an inception cohort. Rheumatology. 2010;49:1483-9.

25. Horiuchi AC, Pereira LHC, Kahlow BS, Silva MB, Skare TL. Rheumatoid arthritis in elderly and young patients. Rev Bras Reumatol Engl Ed. 2017;57: 491-4.

26. Soubrier M, Mathieu S, Payet S, Dubost JJ, Ristori JM. Elderly-onset rheumatoid arthritis. Jt Bone Spine. 2010;77:290-6.

27. Onishi S, Iwmoto M, Minota S. Management of elderly-onset rheumatoid arthritis. Nihon Rinsho Men'eki Gakkai kaishi = Japanese journal of clinical immunology. 2010; 33:1-7.

28. Aletaha D, Smolen JS. Diagnosis and management of rheumatoid arthritis: a review. JAMA. 2018;320: 1360-72.

29. Cho S-K, Sung Y-K, Choi C-B, et al. Do patients with elderly-onset rheumatoid arthritis have severe functional disability? Semin Arthritis Rheum. 2012;42:23-31.

30. Tinedo MAT, Moratalla CP, Torres JRQ. Elderlyonset rheumatoid arthritis (Eora): differences according to clinical debut and serological positivity. Ann Rheum Dis. 2019;78:1632-1632.

31. Alvarez-Rodriguez L, Lopez-Hoyos M, Beares I, et al. Toll-like receptor 4 gene polymorphisms in polymyalgia rheumatica and elderly-onset rheumatoid arthritis. Clin Exp Rheumatol. 2011;29: 795-800.

32. Sugihara $\mathrm{T}$, Ishizaki $\mathrm{T}$, Hosoya $\mathrm{T}$, et al. Structural and functional outcomes of a therapeutic strategy targeting low disease activity in patients with elderly-onset rheumatoid arthritis: a prospective cohort study (CRANE). Rheumatology. 2015;54: 798-807.

33. Salvagno GL, Sanchis-Gomar F, Picanza A, Lippi G. Red blood cell distribution width: a simple parameter with multiple clinical applications. Crit Rev Clin Lab Sci. 2015;52:86-105.

34. Arbel Y, Weitzman D, Raz R, et al. Red blood cell distribution width and the risk of cardiovascular morbidity and all-cause mortality. A populationbased study. Thromb Haemost. 2014;111:300-7.

35. Chebotareva NV, Bobkova IN, Gulyaev SV. Lateonset rheumatoid arthritis in a patient with successfully treated IgA nephropathy. Ter Arkh. 2017;89:77-9.

36. Combe B, Rincheval N. Early lessons from the recent-onset rheumatoid arthritis cohort ESPOIR. Joint Bone Spine. 2015;82:13-7. 